Depot attenders were more likely to seek GP consultation, usually for physical complaints, even when noted to be suffering from active psychosis. Three per cent of depot group consultations led to a blood pressure measurement, compared with $8 \%$ of control group consultations $(P<0.005)$.

Depot neuroleptics are associated with weight gain (a correlate for hypertension), and orthostatic hypotension. Both are indications for monitoring blood pressure. The closure of large psychiatric hospitals has led to increasing numbers of patients with mental illness being treated in a community setting. For some patients depot administration is the only point of contact with the mental heath services.

The institution of blood pressure monitoring on depot clinic attendance provides an opportunity to meet Health of the Nation guidelines for an underprivileged group of patients.

SION ROBERTS, 2 The Drtve, Claybury Hospital, Woodford Bridge, Essex IG8 8BY and DAVID STURGEON, University College Hospital, Gower Street, London WCl

\section{Families and twins with schizophrenia}

Sir: At the Institute of Psychiatry we are carrying out research into genetic and environmental variables which contribute to schizophrenia. As part of this research project, we are carrying out neuropsychological assessments along with magnetic resonance imaging (MRI) of families in which two or more individuals suffer from schizophrenia. We are also studying twin pairs in which one or both have schizophrenia.

This work is highly dependent for its success on the ascertainment of such families and twin pairs. We would be very pleased to be put in touch with any such families or twin pairs that have come to the attention of readers of the Bulletin. We would then personally get in touch with the members of the families and explain the detalls of the project to them. After informed consent, we hope to carry out neuropsychological assessments as well as MRI scans on the well and ill family members.

Please write to or telephone Dr T. Sharma who would be very happy to further explain the project. He can be contacted on 0719193342 , 24-hour bleep: 081812 2564, fax: 071701 9044: Department of Psychological Medicine, Institute of Psychiatry, DeCrespigny Park, Denmark Hill, London SE5 8AF

TONMOY SHARMA and ROBIN MURRAY, Institute of Psychiatry, London SE5 8AF 